\title{
Gerry L’Étang
}

Maître de conférences en anthropologie, Université des Antilles-Guyane.

(2000)

\section{"VINI WÈ KOULI-LA. Anthropologie d'une chanson créole"}

\author{
Un document produit en version numérique par Jean-Marie Tremblay, bénévole, \\ professeur de sociologie au Cégep de Chicoutimi \\ Courriel: jean-marie_tremblay@uqac.ca \\ Site web pédagogique : http://www.uqac.ca/jmt-sociologue/ \\ Dans le cadre de: "Les classiques des sciences sociales" \\ Une bibliothèque numérique fondée et dirigée par Jean-Marie Tremblay, \\ professeur de sociologie au Cégep de Chicoutimi \\ Site web: http://classiques.uqac.ca/ \\ Une collection développée en collaboration avec la Bibliothèque \\ Paul-Émile-Boulet de l'Université du Québec à Chicoutimi \\ Site web: http://bibliotheque.uqac.ca/
}




\section{Politique d'utilisation de la bibliothèque des Classiques}

Toute reproduction et rediffusion de nos fichiers est interdite, même avec la mention de leur provenance, sans l'autorisation formelle, écrite, du fondateur des Classiques des sciences sociales, Jean-Marie Tremblay, sociologue.

Les fichiers des Classiques des sciences sociales ne peuvent sans autorisation formelle:

- être hébergés (en fichier ou page web, en totalité ou en partie) sur un serveur autre que celui des Classiques.

- servir de base de travail à un autre fichier modifié ensuite par tout autre moyen (couleur, police, mise en page, extraits, support, etc...),

Les fichiers (.html, .doc, .pdf., .rtf, .jpg, .gif) disponibles sur le site Les Classiques des sciences sociales sont la propriété des Classiques des sciences sociales, un organisme à but non lucratif composé exclusivement de bénévoles.

Ils sont disponibles pour une utilisation intellectuelle et personnelle et, en aucun cas, commerciale. Toute utilisation à des fins commerciales des fichiers sur ce site est strictement interdite et toute rediffusion est également strictement interdite.

L'accès à notre travail est libre et gratuit à tous les utilisateurs. C'est notre mission.

Jean-Marie Tremblay, sociologue

Fondateur et Président-directeur général, LES CLASSIQUES DES SCIENCES SOCIALES. 
Cette édition électronique a été réalisée par Jean-Marie Tremblay, bénévole, professeur de sociologie au Cégep de Chicoutimi à partir de :

Gerry L’Étang

“VINI WÈ KOULI-LA. Anthropologie d'une chanson créole.”

Un article publié dans l'ouvrage sous la direction de Jean-Luc Bonniol, Gerry L'Étang, Jean Barnabé et Raphaël Confiant, Au visiteur lumineux. Des îles créoles aux sociétés plurielles. Mélanges offerts à Jean Benoist, pp. 659-671. PetitBourg, Guadeloupe : Ibis Rouge Éditions, GEREC-F/Presses universitaires créoles, 2000, 716 pp.

[Autorisation formelle accordée par l'auteur le 20 mars 2008 de diffuser cet article dans Les Classiques des sciences sociales.]

Courriel : gletang@martinique.univ-ag.fr

Polices de caractères utilisée :

Pour le texte: Times New Roman, 14 points.

Pour les citations : Times New Roman, 12 points.

Pour les notes de bas de page : Times New Roman, 12 points.

Édition électronique réalisée avec le traitement de textes Microsoft Word 2004 pour Macintosh.

Mise en page sur papier format : LETTRE (US letter), 8.5’’ x 11’')

Édition numérique réalisée le 5 juillet 2008 à Chicoutimi, Ville de Saguenay, province de Québec, Canada. 


\section{Gerry L’Étang}

Maître de conférences en anthropologie, Université des Antilles-Guyane.

\section{"VINI WÈ KOULI-LA.}
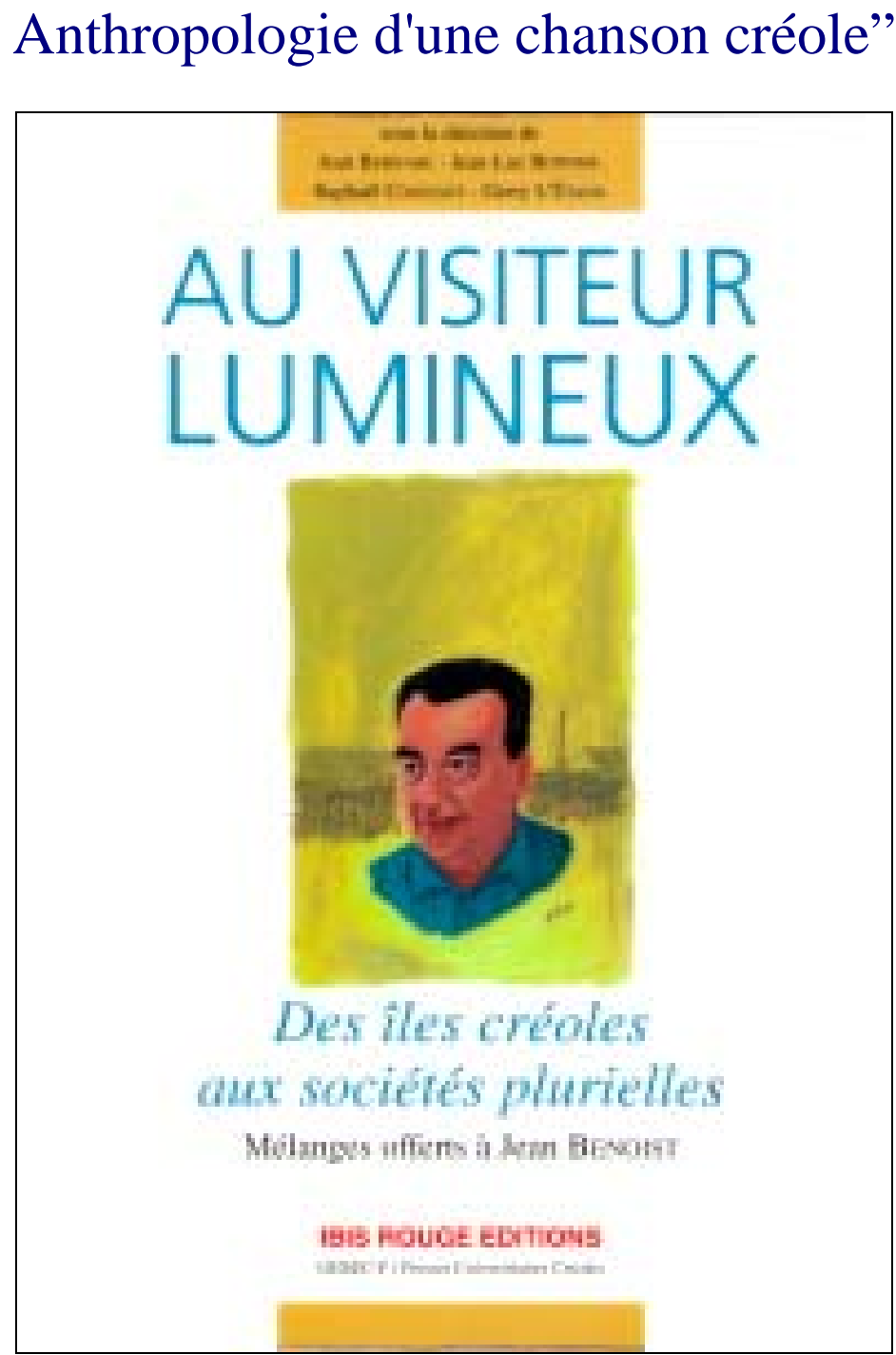

Un article publié dans l'ouvrage sous la direction de Jean-Luc Bonniol, Gerry L'Étang, Jean Barnabé et Raphaël Confiant, Au visiteur lumineux. Des îles créoles aux sociétés plurielles. Mélanges offerts à Jean Benoist, pp. 659-671. PetitBourg, Guadeloupe : Ibis Rouge Éditions, GEREC-F/Presses universitaires créoles, 2000, 716 pp. 


\section{Gerry L’Étang}

Maître de conférences en anthropologie, Université des Antilles-Guyane.

“VINI WÈ KOULI-LA. Anthropologie d'une chanson créole”.

Un article publié dans l'ouvrage sous la direction de Jean-Luc Bonniol, Gerry L'Étang, Jean Barnabé et Raphaël Confiant, Au visiteur lumineux. Des îles créoles aux sociétés plurielles. Mélanges offerts à Jean Benoist, pp. 659-671. PetitBourg, Guadeloupe : Ibis Rouge Éditions, GEREC-F/Presses universitaires créoles, 2000, 716 pp.

C'est en situant les descendants des immigrants asiatiques dans la société globale que nous devons procéder. Car ils sont, depuis leur arrivée, en interaction constante avec elle. Même lorsqu'elle les rejette, elle les transforme et ils l'influencent.

(Benoist, 1976 : 167)

Le présent article trouve son origine dans un incident, un après-midi d'octobre chez un informateur, dévot hindou qui nous contait l'histoire de Madévilen, dieu à cheval, guerrier flamboyant à la geste toute de bruit et de fureur. Dans un coin de la pièce, une radio diffusait un programme musical. Malgré l'enregistrement de notre entretien, l'homme avait insisté pour laisser courir une émission d'airs créoles traditionnels.

C'est alors que résonna la voie gouailleuse de Léona Gabriel ${ }^{1}$. L'homme se leva précipitamment et éteignit le poste : « je ne veux pas entendre cette chanson,

1 Léona Gabriel (1891-1971), née à la Martinique, arriva à Paris dans les années vingt, après avoir séjourné en Guyane et à Panama. Elle y épousa en 1928 le célèbre compositeur français Léo Daniderff (Ferdinand Niquet), qu'elle quitta quelques mois plus tard. Elle enregistra de nombreux disques et est considérée comme la chanteuse de référence de la musique traditionnelle martiniquaise. Servie par une mémoire remarquable, elle restitua, avec des arrangements qui lui sont propres, une partie du patrimoine musical de Saint-Pierre. Dans un ouvrage qui réunit son répertoire (ses compositions et des morceaux issus du folklore pierrotin), elle déclare, concernant les textes de ces chansons : «Ce 
elle m'a fait trop mal ! ». Il retourna s'asseoir pour rester de longues minutes sans mot dire, perdu dans des réminiscences, marque par le ressentiment ; puis, devant notre air interloqué, entreprit de nous raconter une tout autre histoire :

C'était il y a longtemps. Au temps des premiers orchestres haïtiens, à l'époque de la Bananeraie, de l'Impériale Paillote, du Dancing Palace... de ces grands bals qui rassemblaient jusqu'à 2000 danseurs.

Ce soir-là, j'avais levé une petite chabine ${ }^{2}$ avec des yeux verts et une peau d'une douceur.. Nous avions commencé à danser avec Tropicana, puis un orchestre local avait pris la suite. Malgré d'autres sollicitations, la chabine ne dansait qu'avec moi, et aux regards jaloux des autres types, il était clair qu'elle était pour moi. C'est alors que l'orchestre s'est mis à jouer cette chanson. Je ne sais pas ce qui m'a pris : par panique ou par bravade, je ne sais plus, j'ai voulu danser, danser encore. La chabine accepta. Mais les gens commençaient à sourire, à plaisanter. Et puis un salaud a gueulé Ladjé'y, kouli-a, ladjé'y ! pa ta'w ${ }^{3}$ Toute la salle s'est mise à rire. La chabine n'a pas supporté elle m'a planté là. Je ne l'ai jamais revue...

J'ai quitté le bal peu après. Des bougres s'étaient mis àm'injurier, à me traiter de Coolie ... Le mot, à l'époque, résonnait dans ma tête comme une bombe atomique ...

La déconvenue de notre informateur, la survenue dans un bal d'une chanson stigmatisant son groupe ethnique, l'utilisation de cet air par ses rivaux pour empêcher une entreprise de séduction pourtant bien engagée, tout ceci nous conduisit à interroger ladite chanson. Dans quel arrière plan anthropo-historique s'enchâsse-telle, de quels discours se fait-elle l'écho?

ne sont que des récits vrais de certaines de nos coutumes, de nos mœurs, de nos traditions ; Ce sont des récits sans fard, sans artifice, un pur métal sans alliage, des récits tout nus » (Gabriel-Soïme, 1966 : 14).

2 Le chabin (ou la chabine) désigne à la Martinique un phénotype particulier présentant, pour reprendre le mot de Michel Leiris, " une combinaison paradoxale de traits des races noire et blanche » $(1955: 161)$ : cheveux blonds et crépus, peau claire et traits négroïdes, etc. En rapport avec cette singularité, on prête au chabin un caractère instable voire querelleur (mové chaben). La chabine, elle, est davantage réputée pour l'attraction qu'elle suscite, quand la combinaison en question est jugée harmonieuse.

3 « Lâche-la, coolie, lâche-la ! Elle n'est pas pour toi ! » 
La chanson en question fut enregistrée pour la première fois à Paris, chez Polydor, en juillet 1931. La capitale française ballait alors au rythme de la biguine, découverte quelques années auparavant au célèbre Bal nègre du 33 rue Blomet et qui s'était imposée comme musique des chaudes nuits parisiennes à l'arrivée, en 1929, du clarinettiste martiniquais Alexandre Stellio ${ }^{4}$ et de son orchestre, le Stellio's Band, composé, entre autres, d'Ernest Léardée et d'Archange Saint-Hilaire.

En 193 1, Stellio, en rupture avec son orchestre historique pour cause de mésentente sur la répartition des bénéfices (Meunier / Léardée, 1989 : 160), rassembla une nouvelle formation, L'orchestre Stellio de l'Exposition coloniale, attraction de l'exposition du même nom fréquentée par des millions de visiteurs du 6 mai au 15 novembre 1931 (Meunier, 1994 : 10). L'orchestre comptait une des plus fameuses chanteuses martiniquaises de l'époque, Léona Gabriel, revenue dans le milieu antillais après un détour par les cabarets français et qui apportait la chanson : Vini wè kouli-a.

Cette biguine qui connut dès sa sortie un certain succès, allait être relancée à la Martinique lors de son réenregistrement en 1962 par Léona Gabriel, accompagnée, cette fois, par l'Orchestre Printemps jazz d'Archange Saint-Hilaire. Elle reparut avec quelques modifications apportées au titre et au refrain, et fut alors reprise par différents orchestres de bals. C'est probablement à l'une de ces occasions que notre informateur connut sa mésaventure.

Cet air est considéré depuis comme un chef-d'oeuvre de la chanson créole martiniquaise. Les versions de 1931 et de 1962 ont été rééditées sur support C.D., dans le cadre de compilations à vocation patrimoniale (Stellio, 1994 ; Best of,

4 Alexandre Stellio (Fructueux Alexandre, 1885-1939) naquit à la Martinique, vécut à Saint-Pierre et séjourna en Guyane avant de retourner dans son île natale pour s'y imposer comme le meilleur clarinettiste d'alors. Son installation dans la capitale française en 1929 fit de lui « le créateur de la biguine à Paris ». En 10 ans (jusqu'à qu'il s'écroule sur scène victime d'une embolie), il y enregistra au moins de 128 faces et des dizaines de chansons (ses créations et des airs de Saint-Pierre). Il est réputé pour avoir été « L'étoile noire de la musique créole » (Boulanger, 1991 : 5). 
1997), et son refrain est réapparu récemment dans un pot-pourri d'airs traditionnels réorchestrés au goût du jour (zouk) par « les stars de la musique antilloguyanaise » (Ethnikolor, sans date).

Attribuée à Léona Gabriel (paroles et musique), cette chanson provient en réalité du vieux fond musical de Saint-Pierre d'avant l'éruption de 1902. Sa création est probablement contemporaine de l'immigration indienne à la Martinique (18531883). Elle expose d'ailleurs les mésaventures d'une femme créole malmenée par un immigrant indien :

premier couplet

Nonm-lan sòti lòt bò péyi'y, I pasé dlo vini isi, Tout moun té ka pran li pou moun, Pandan tan-an sé vakabon (bis).

Refrain

Woy ! Vini wè kouli-a, woy Kouli-a, kouli-a, woy!

Ba li lè pou li pasé, Pou li fè kout twotwè li kanmenm Woy ! Vini wè kouli-a, woy Kouli-a, kouli-a, woy!

Ba li lè pou li pasé, Pou li peu chanjé de konduit. deuxième couplet Mwen fè si mwa dan le ménaj, Mi tout lajan nonm-lan ban mwen I ba mwen di fran man ba bòn mwen, Fo mwen mété sen fran asou'y.

troisième couplet

Mwen fè twa mwa de maladi, Mi tout rumèd nonm-lan ban mwen, Mi tout mèdsen nonm-lan ban mwen : I ba mwen an nonm pou swanyé mwen.

Refrain 
Woy ! Vini wè kouli-a, woy

Kouli-a, kouli-a, woy!

Ba li lè pou li pasé,

Pou li fè kout twotwè li kanmenm

Woy ! Vini wè kouli-a, woy

Kouli-a, kouli-a, woy!

Ba li lè pou li pasé,

Pou li peu chanjé de konduit ${ }^{5}$.

Nous ne retiendrons de ce texte que le plus significatif, c'est-à-dire son premier couplet, l'exposé d'une désillusion, ainsi que son refrain dont l'expression Kouli (scansion renforcée par l'onomatopée Woy !) fait sens. Le reste est anecdotique.

5 Premier couplet

L'homme a quitté son pays d'un autre bord,

Il a passé J'eau pour venir ici,

Tous le prenaient pour quelqu'un,

Ce n'était en fait qu'un vagabond (bis).

Deuxième couplet

J'ai fait six mois dans le ménage,

Voyez quel argent il m'a donné :

Il m'a donné dix francs pour ma bonne,

J'ai dû en sortir cinq de ma poche.

Troisième couplet

J'ai fait trois mois de maladie,

Voyez quel remède il m'a donné,

Voyez quel médecin il m'a donné

Il m'a donné un homme pour me soigner.

\section{Refrain}

Woy ! Venez voir le coolie, woy

Le coolie, le coolie, woy!

Laissez-le passer,

Afin qu'il fasse quand même son coup de trottoir.

Woy! Venez voir le coolie, woy

Le coolie, le coolie, woy!

Laissez-le passer,

Afin qu'il puisse changer de conduite.

(version de 1931) 
L'incipit de cette chanson, son premier couplet, constitue un étonnant résumé de ce que représenta l'accueil des Indiens à la Martinique : enthousiasme puis rejet. L'enthousiasme trouve son fondement contradictoire dans l'utilisation escomptée de cette immigration par la plantocratie békée et dans la tradition d'hospitalité de la population martiniquaise d'alors.

Suite à l'abolition de l'esclavage (1848), les planteurs, menacés dans leurs intérêts par les revendications salariales des nouveaux libres, obtinrent en 1852 du futur Napoléon III l'organisation d'une immigration sous contrat. Dans ce contexte, l'immigration indienne fut préférée à la seule immigration massive organisée parallèlement, l'immigration congo ${ }^{6}$, en raison du risque politique que cette dernière présentait. « L'Indien, espérait-on, n'étant ni de la même race ni de la même religion et ne parlant pas la même langue, éviterait tout rapprochement avec les Noirs et ferait cause commune avec les propriétaires » (Lugsor, 1980 : 39).

Dans cet ordre d'idée parut dans le Moniteur de la Martinique du 4 février 1855, une vibrante apologie de l'immigration indienne :

Universellement, on déclare que l'immigration indienne est la meilleure, qu'il n'y a même que l'immigration indienne qui soit bonne. L'Indien (est) un élément qui ne peut en aucun cas être l'objet de craintes (...) Il nous est impossible (...) de nous empêcher de regarder comme un avantage sérieux des coolies de ne jamais pouvoir servir de prétexte à l'embrasement de passions mai éteintes. Ils n'arriveront jamais à la Martinique (...) que comme cultivateurs; ils seront nuls en dehors du travail, neutres alors même que le cercle s'élargirait pour eux. Il n'est pas une mutinerie d'habitation, une grève qui pourrait compter sur eux. En dehors de tous, ils sont précieux pour tous. Peut-on dire en autant des autres immigrants?

6 De 1857 à 1862, 10521 engagés africains embarquèrent des ports du CongoKinshasa et du Congo-Brazzaville pour la Martinique (25 509 Indiens arrivèrent, eux, entre 1853 et 1883). À la faveur d'une communauté d'origine et de phénotype avec les Noirs créoles, ils s'intégrèrent rapidement. Les Congos et leurs descendants furent au côté des autochtones dans toutes les luttes contre J'arbitraire colonial, qu'il s'agisse de l'Insurrection du Sud (1870) ou de la Grève du François (1900), confirmant les craintes des engagistes quant à leur insoumission à l'ordre plantocratique. 
Ignorante des motivations ayant inspiré l'appel à cette immigration, la société noire créole réserva, dans un premier temps, le meilleur accueil aux arrivants, observant en cela une tradition d'hospitalité soulignée par les écrivains voyageurs qui fréquentèrent l'île au XIXe siècle ${ }^{7}$. Une lettre du Gouverneur en date du 27 mai 1853, signale que les 313 premiers Indiens débarqués par l'Aurélie furent accueillis «avec beaucoup de cordialité ; ils (les Noirs) les accablent de leur curiosité et de leur générosité en leur faisant de petits présents » (cité par Renard, 1973 : 229). Ailleurs, dans le Journal officiel de la Martinique du 21 octobre 1858, « les coolies arrivés par le Réaumur sont jugés jeunes, beaux et font l'admiration de tout le monde ».

Plusieurs écrits des premières années d'immigration s'accordent sur l'humanité des Indiens, comme pour l'immigrant de la chanson, à propos duquel il est mentionné dans le premier couplet: Tout moun té ka pran li pou moun: "Tous le prenaient pour quelqu'un » (une personne, un homme).

Cet enthousiasme n'allait pas durer. Après quelques années, il apparut aux travailleurs autochtones que l'arrivée de milliers de contractuels instrumentalisés par les planteurs aux fins d'une saturation de l'offre de travail et d'un maintien des bas salaires, desservait leur cause. Cette manœuvre était d'autant plus frustrante qu'elle s'inscrivait, après l'euphorie de l'abolition, dans un mouvement général de reprise en main de la force de travail coloniale, dès les débuts de la Il, République puis avec Louis Napoléon Bonaparte, sous cette même République et tout au long du Second Empire.

Le retour de la République après la débâcle de Sedan allait toutefois aggraver l'antagonisme entre autochtones et arrivants. Revenus après 1870 dans le débat politique local, les schoelchéristes, soucieux de mettre un terme a une immigration qui perdurait, s'en prirent à la valeur de l'Indien. Ainsi Victor Schœlcher luimême remarquait en 1880, qu'« il est trop vrai que les immigrants sortent de la lie de la population indienne, que leur contact a la plus mauvaise influence sur les mœurs des campagnes des Antilles » (rééd. 1979, 1 : 283).

7 On consultera notamment sur ce point Lafcadio Hearn, ses Esquisses martiniquaises (trad. 1924) ou ses Contes des Tropiques (trad. 1927). 
Le discours de Schoelcher à l'égard des engagés indiens est en réalité ambivalent. Il oscille entre la dénonciation du sort qui leur fut réservé et la stigmatisation de leur dépravation. Dans l'ouvrage Polémique coloniale qui réunit les principaux articles qu'il consacre à cette immigration, l'auteur manifeste son apitoiement pour ces engagés maintenus « dans la condition de serfs de la glèbe (...), sans aucune garantie contre les abus du pouvoir dominical (ibidem, I : 279), victimes d'une «terrible mortalité qui les décime» (Ibid., I : 269), "l'immigration ayant consommé presque autant de créatures humaines qu'en consommait l'esclavage » (Ibid., II : 225).

Dans le même temps, les immigrants lui apparaissent comme des «mercenaires mal choisis (qui exercent) dans les campagnes, une influence démoralisatrice » (Ibid., I : 269). Il va même jusqu'à suggérer l'existence de pratiques homosexuelles communes chez ces « aides si dangereux » (Ibid., I : 269) - accusation alors infamante - et à souhaiter, pour ceux qui se vengeaient de leurs engagistes en incendiant les récoltes, « l'établissement d'un bagne installé dans un ponton, sur rade de Fort-de-France » (Ibid., I : 277).

Les contradictions du discours de Schœlcher ne sont qu'apparentes. Elles procèdent en fait d'une double condamnation de l'immigration indienne. Pour l'auteur en effet, cette dernière est foncièrement dommageable : d'abord parce qu'elle consume les vies de ceux qui en sont l'objet, ensuite parce qu'elle présente des risques de pollution morale pour ceux amenés à la côtoyer. Autrement dit, l'immigration est préjudiciable autant à l'Indien qu'au Créole.

L'immoralité qu'il prête aux immigrants n'en pose pas moins question. Schœlcher - dont le buste orne un carrefour de Pondichéry - fut relativement connaisseur des réalités indiennes. Il eut, en sa qualité de leader des parlementaires républicains des colonies, à intervenir dans les questions qui agitèrent les Établissements français dans l'Inde. Il s'éleva notamment contre la condition réservée aux intouchables 8 :

8 À en croire M. About, en 1884, « les droits du paria dans l'Inde française sont approximativement les mêmes que ceux du porc dans la banlieue de Paris ». 
victimes d'une « législation religieuse absolument barbare, où l'on voit des parias, des créatures humaines, mis au rang des animaux impurs du mosaïsme, véritables proscrits à l'intérieur, tellement avilis par la théorie théocratique des castes qu'il ne leur est pas même permis d'entrer dans une pagode pour y faire leurs prières et que le moindre contact de leurs vêtements est réputé une souillure » (Ibid., II : 161).

Manifestement, la compassion de l'humaniste, préoccupé dans le cas de l'Inde par l'abjection dans laquelle étaient tenus les ressortissants des plus basses castes, rencontra peu d'écho chez le politicien de la Martinique, soucieux exclusivement des intérêts de ses électeurs, ces « laboureurs créoles qui n'aiment pas le contact des coolies, dont la concurrence a fait baisser les salaires » (Ibid., I : 273).

En raison de son poids moral autant que politique, Schœlcher prit une part non négligeable dans la transformation de l'Indien, de victime en coupable. Ce qui eut pour conséquence de conforter les planteurs dans leur stratégie d'opposition d'un groupe à un autre et d'occulter leur responsabilité dans cette manœuvre.

L'idée que les Indiens constituaient une lie d'humanité dépassait cependant le milieu des politiciens républicains. Ainsi, l'essayiste P. Leroy-Beaulieu écrivait en 1874 :

L'immigration de coolies sur une très grande échelle est peut-être encore plus dangereuse que le maintien de l'esclavage, (eu égard à) la corruption asiatique que ces aventuriers appartenant à la lie (de la) société indienne inoculent aux sociétés européennes (et en raison de) l'instabilité qui résulte de cette vaste population flottante que rien n'attache à la terre qu'elle cultive (1874 : 574).

Nonobstant les qualités qu'ils prêtaient initialement aux originaires de l'Inde, certains planteurs alimentèrent même la suspicion sur la nature de l'Indien. L'usinier conservateur antillais Ernest Souque, tout en jugeant l'immigration indienne indispensable, considérait en 1884 que cette dernière, « qu'elle se recrute à Pondichéry ou à Calcutta, est composée, presque en totalité du rebus de l'Inde. Elle est indolente, possède les vices des races dégénérées, se nourrit difficilement, sa reli- 
gion lui imposant souvent l'obligation de ne pas manger de viande »(cité par Schœlcher, rééd. 1979, II : 232).

Il faut dire que les propriétaires terriens s'étaient heurtés à certains contractuels qui, rétifs au sort qui leur était réservé, embrasaient les cannaies et les cases à bagasse. Les dizaines de plaintes émanant des planteurs, les fréquentes condamnations pour pyromanie attestent de l'utilisation de l'incendie comme mode de vengeance indien, comme réaction des engagés aux abus dont ils étaient victimes. Il ressort de la ventilation des faits délictueux recensés par le journal officiel de la colonie pour les années 1855 à 1891, qu'il y eut au moins 166 cas d'incendies commis au cours de cette période par des Indiens (Sméralda-Amon, 1996 : 357).

En répercussion aux discours cités et dans une situation politique, économique, culturelle et sociale défavorable aux engagés et aux premières générations de leur descendance, fleuriront des productions de l'oraliture populaire créole flétrissant les Indiens, au rang desquelles la chanson Vini wè kouli-a ou le proverbe Tout kouli ni an kout twotwè pou i fè ${ }^{9}$.

Ces deux productions oralituraires sont d'ailleurs liées, dans la mesure où la seconde apparaît contenue dans la première. Le refrain invite en effet à « laisser passer le Coolie (en fait à éviter un ivrogne titubant), afin qu'il fasse quand même son coup de trottoir ». Le coup de trottoir en question, sanction de l'Indien pour son inconduite à l'endroit de la Créole, désigne sa chute dans le caniveau sous l'effet de l'alcool.

Le passage des engagés d'une société hindoue marquée par la tempérance a un pays où le rhum imbibait profondément les modes de vie populaires, fut pour certains d'entre eux catastrophique. D'autant que la surconsommation d'alcool était encouragée par des planteurs peu scrupuleux qui payaient pour partie en tafia (donc à moindre frais) leurs contractuels. Concernant les ravages de l'alcoolisme, le médecin du Marie-Laure, convoi de rapatriés en partance pour l'Inde, note :

9 Ce proverbe (ou sa variante : tout kouli ni an kout dalo pou i fè) signifie : « Tout Indien se retrouvera un jour ou l'autre dans le caniveau ». 
La vue de nos nouveaux passagers était loin de rappeler nos premiers compagnons de voyage ; au lieu de ce convoi plein de jeunesse et de santé que nous avions pris dans l'Inde, nous avions sous les yeux des hommes dont le plus grand nombre étaient vieux et maigres (...). Leur physionomie, pour beaucoup, traduisait le dégoût et la haine (...). Presque tous les hommes présentaient cette pâleur si caractéristique chez les ivrognes (cité par Singaravélou, 1987, I : 160).

Dans ces circonstances, une disposition à l'éthylisme fut imputée à l'Indien : « d'après Cornillac, l'Indien, à peine débarqué, deviendrait un ardent buveur de tafia et rendrait des points aux nègres sous le rapport de l'ivrognerie (Corre, 1889 : 152) ».

Il convient encore de signaler un second sens à ce proverbe, lequel, plus tardif, date de la première moitié du XXe siècle. A l'issue des retours en Inde (le dernier convoi quitta l'île en 1900), se retrouvèrent au dépôt de l'immigration sis à Fortde-France quelques dizaines d'Indiens qui attendaient là un improbable navire de rapatriement, ou encore qui, venus embarquer, s'étaient ravisés et avaient décidé de rester à la Martinique. Loin des Habitations, ils vivaient d'expédients et constituaient un souci pour le Conseil général (qui avait en charge le dépôt) et la municipalité. Cette dernière les affecta alors au nettoiement de la ville.

Ce groupe de balayeurs indiens, renforcé d'apports successifs en provenance des plantations à mesure que s'étendait le chef-lieu, se vit attribuer l'exclusivité d'une tâche méprisée ${ }^{10}$. Et le proverbe de s'enrichir d'une nouvelle acception : « tout Indien se retrouvera un jour ou l'autre balayeur de trottoir ». En fait, dans un cas comme dans l'autre, l'expression énonce une malédiction.

Cette dépréciation générale de l'Indien allait s'exacerber au travers de l'appellation créole qui le stigmatisera : Kouli. L'expression, probablement d'origine tamoule (kuli), signifie originellement salaire et par extension salarié. Elle fut utilisée par les Anglais puis par les Français en Extrême-orient (Inde, Chine, etc.) pour qualifier un ensemble varié de travailleurs non spécialisés aux revenus pré-

10 Sur l'histoire orale du dépôt et sur sa délocalisation au lieu-dit Au-Béro, on consultera Jean-Pierre Arsaye (1998). 
caires : employés aux travaux pénibles, dockers, manoeuvres, tireurs de pousse, journaliers agricoles, ouvriers, etc. 11

L'emploi du mot dans cette acception est encore fréquent dans certaines régions de l'Inde. Il apparaît en conséquence dans la littérature indienne, dans le roman Coolie par exemple, qui décrit le parcours chaotique d'un enfant du Punjab, tour à tour domestique, commis, ouvrier exploité des filatures de Bombay, etc. (Anand, trad. 1947). On retrouve l'utilisation de ce vocable avec un sens équivalent dans certains essais sociologiques comme Femmes coolies en Inde, consacré aux portefaix féminines du grand marché de Pune (Poitevin / Rairkar, 1994).

En rapport avec la condition de travailleurs des engagés, le terme fut usité par les Européens de l'Inde anglaise et des Établissements pour désigner l'immigration indienne dans son ensemble. Les expressions Coolie Trade et Coolie Ship en attestent. De même, un provicaire apostolique des Missions étrangères, rédacteur en 1863 à Pondichéry d'une Grammaire française-tamoule, justifie cette œuvre par l'argument suivant : « l'émigration des coulis, pour nos autres colonies, la rend indispensable à bien des Européens qu'elle emploie, et ce besoin se fait sentir jusqu'à Bourbon et à la Martinique »(Anonyme, 1863 : 5).

Parfois cependant le vocable semble caractériser un type spécifique d'immigrants. Le médecin du Siam (bateau introducteur d'Indiens) l'emploie dans le sens particulier de «cultivateur démuni », par opposition aux engagés moins déshérités. C'est ce qui ressort du passage mentionnant que :

11 Louis Frédéric signale la possibilité d'une autre étymologie, « le mot venant peut-être de l'ancien nom d'une caste de travailleurs de la région de Bombay, appelée aussi Kulî », (Frédéric, 1987 : 647).

Le poète indo-mauricien Khal Torabully note encore à propos du même mot : «Les Kulis sont les habitants de Kula, dans la région indo-gangétique, peuple semi-nomadique, rompu aux travaux agricoles intensifs. Le mot Coolie, certainement celui qui aura le plus marqué le vocabulaire colonial de l'après-esclavage, subit un glissement sémantique. De l'habitant de Kula au statut de porteur, et, plus tard, à tout va-nu-pieds, il n'y a qu'une... réduction de son existence en tant qu'être humain. Il devient métonymie : petites mains ou bras... Coolie, portion congrue, paria parmi les harijans (intouchables), bête de somme, presque objet - ce qui n'est pas sans conséquence sur sa prise de parole. Est Coolie celui qui travaille et ne parle pas », (Torabully, 1996 : 59). 
Les Indiens qui émigrent, appartiennent presque tous à la classe la plus malheureuse, à celle pour qui les moyens d'existence sont souvent un problème ; ce sont presque tous des cultivateurs (coolis) ; cependant, il se trouve parmi eux quelques individus de castes plus relevées (Leclerc, 1860 : 106).

Le mot subira à la Martinique un glissement sémantique. Alors qu'en Inde il caractérisait principalement un état professionnel marqué par la précarité, et secondairement l'émigration de travail partant de ce pays, il s'imposera dans la colonie comme ethnonyme désignant tout originaire de l'Inde. Il y prendra en outre une connotation dépréciative. On le retrouvera donc, en rapport avec le statut dégradant des nouveaux arrivés, dans toutes les formules créoles avilissant les Indiens.

La valeur péjorative du terme apparaîtra encore implicitement dans l'expression dérivée chapé-kouli (ou échapé-kouli), désignant l'individu issu du brassage d'éléments d'origine indienne et africaine mais dont l'ascendance indienne reste repérable du point de vue du phénotype. Caractériser le produit de ce métissage comme ayant en quelque sorte "échappé à la race des coolies », c'est sousentendre qu'en vertu de ce croisement, il l'a échappé belle !

Le mot figure de façon répétitive dans la chanson et a valeur d'injure. Il y est convié à « venir voir le coolie » (vini wè kouli-a), soit à constater l'infamie de ce dernier. La version de 1962 invite même à le huer (vini kriyé koulia). Car loin d'être quelqu'un, il se révèle un vakabon, terme désignant non pas l'errant (le vagabond) mais le coupable de vakabonajri : faits de voyoucratie et autres actes d'immoralité.

Et comme cela ne suffisait pas, le refrain de 1962 abandonne la référence au coolie-ivrogne pour un personnage plus répugnant encore : le cooliepouilleux, duquel on est prié de s'écarter, « afin qu'il se débarrasse de ses poux blancs » (Ba li lè pou li pasé, pou li jété sé pou blan'y lan).

Le thème du coolie-pouilleux apparaîtra dans une des rares mentions de l'Indien dans la littérature martiniquaise du début du siècle, sous la plume de la ro- 
mancière Irmine Romanette et dans une suite de caractérisations des groupes ethniques et sociaux composant la Martinique d'alors. Le passage a pour cadre l'ossuaire dressé à Saint-Pierre suite à la Catastrophe :

Dans l'ossuaire du souvenir, élevé au pied du Morne Abeille à la mémoire des disparus, les squelettes sont confondus. Ceux que des fouilles ramènent au jour, attendent pêle-mêle d'être déposés dans le trou d'ombre et restent exposés aux regards sur la dalle de marbre, proposant ainsi une angoissante énigme aux passants. Réponds ! Qui suis-je ? La servante noire ou la matrone blanche? Le travailleur serf ou le planteur féodal ? Le coolie pouilleux du marché ou le richissime commerçant de la Place ? La frémissante maîtresse ou l'épouse acariâtre ? (cité par Corzani, 1999 : 81).

Au final, et en résonance d'un propos général de détraction de l'Indien, c'est un portrait singulièrement sordide de ce dernier que brosse cette chanson : étranger imposteur, pingre, goujat, ivrogne, pouilleux de surcroît ; scélérat sommé de cesser ses frasques, de « changer de conduite ».

Par-delà sa xénophobie et son racisme, ce que nous dit cette chanson, c'est que l'inscription des Indiens dans le processus de créolisation à la Martinique - c'est-àdire de rencontre, d'emmêlement et de croisement des hommes et des cultures s'est faite dans la douleur. Mais la créolisation est une dynamique et son résultat est par nature instable. Les petits-fils d'immigrants indiens bénéficieront d'une évolution favorable.

À l'issue des années soixante, avec la fin de la société d'habitation, à savoir la disparition de la plantation en tant qu'institution structurante de la société martiniquaise, disparurent le cadre et les raisons d'une opposition entre Créoles et originaires de l'Inde, qui trouvait son fondement dans l'instrumentalisation de ces derniers comme briseurs de grève et auxiliaires des planteurs contre le groupe majoritaire.

Parallèlement, la modernité qui allait se mettre en place, à défaut d'être autoproduite et autogérée, se traduisit par la généralisation de l'assistance, l'immigra- 
tion en France, l'urbanisation incontrôlée, l'apparition de media de masse valorisant d'autres configurations culturelles, la déliquescence des traits créoles les plus saillants ou leur folklorisation au travers de leur mise en spectacle par l'appareil touristique ; toutes choses menaçant le Martiniquais d'une « décréolisation compréhensive » (Burton, 1994 : 214). Faute de maîtriser les flux économiques et culturels qui le traversent, il encourt aujourd'hui le risque de se voir zombifié, c'est-à-dire « transformé en un transfusé inactif maintenu en survie par l'assistance et le téléguidage » (Blanquart, 1993 : 157).

Cette mutation est ressentie avec d'autant plus de malaise au plan culturel que l'idéal d'assimilation à la Francité, généralisé aux lendemains de l'abolition et partagé longtemps par l'écrasante majorité de la population, ne semble plus à l'ordre du jour. Paradoxalement, la quasi-concrétisation de l'assimilation advint dans un environnement anti-assimilationniste, en raison de la remise en question de cet idéal par les tenants de la Négritude puis de l'Antillanité et de la Créolité, et eu égard au fait qu'on ne peut être assimilationniste que si l'on n'est pas déjà assimilé.

Longtemps maintenus à l'écart, n'ayant d'autre choix que de cultiver des valeurs qui étaient les leurs et de maintenir leur différence, les originaires de l'Inde entrèrent dans la société post-agricole martiniquaise avec un héritage culturel substantiel. Cet héritage est aujourd'hui revendiqué par une société qui affirme son opposition à l'assimilation totale à la culture dominante et qui, à la recherche d'hétérogénéité en contexte d'identification, incorpore ce qu'elle rejetait jadis.

Cette intégration trouve aussi son fondement dans l'évolution du complexe culturel général, par la dynamique même d'un processus de créolisation qui, en dépit du mépris dont l'Indien était l'objet, fit à la longue de ce dernier un acteur du fait créole, un co-producteur de cette culture.

\section{Références bibliographiques}

ANAND, Mulk Raj, Coolie, Nagel (trad. fr.), Paris, 1947. 
ANONYME, Grammaire française-tamoule, ou les règles du tamoul vulgaire, celles de la langue relevée et celles de la poésie tamoule, par un provicaire apostolique de la congrégation des Missions étrangères, Asian Educational Services, New-Delhi, 1863, rééd. 1984.

ARSAYE, Jean-Pierre, Mémoire d'Au-Béro. Quartier indien de Foyal, GEREC / PUC / Ibis Rouge, Petit-Bourg, Guadeloupe, 1998.

BENOIST, Jean, « Engagés asiatiques et sociétés créoles : quelques conclusions », Actes du XLIIe congrès international des américanistes, Paris, 1976, p. 165-168.

BEST OF, Hurard Coppet, Léona Gabriel, Saint-Hilaire, Jazz antillais, Sully Cally, Collection patrimoine, Fort-de-France, 1997.

BLANQUART, Paul, «L'anticipation caribéenne : nouvel individu et interculturalité », Tyanaba, no 3, Fort-de-France, 1993, p. 153-157.

BOULANGER, Alain, livret de : L'étoile de la musique créole : Stellio et son orchestre antillais, (Compact Dise), Music Merroria, Paris, 1991.

BURTON, Richard, «Penser l'Indianité. La présence indienne dans la réflexion martiniquaise contemporaine », Présences de l'Inde dans le monde, (Gerry L'Étang éd.) PUC / GEREC / L'Harmattan, Paris, 1994, p. 205-216.

CORRE, A., Le Crime en pays créoles. Esquisse d'ethnographie criminelle, Stock, Lyon-Masson, Paris, 1889.

CORZANI, Jack, « La fortune littéraire de la Catastrophe de Saint-Pierre. Entre commémoration et mythification : le jeu des idéologies », in Les Catastrophes naturelles aux Antilles. D'une Soufrière à l'autre (A. Yacou éd.), Karthala-CERC, Paris, 1999, p. 75-97.

ETHNIKOLOR, The Best of Ethnikolor. Dansez les plus grands succès de la musique antillaise... La fête antillaise continue... (Compact Dise), Paris, sans date. 
FREDERIC, Louis, Dictionnaire de la civilisation indienne, Robert Laffont, Paris, 1987.

GABRIEL-SOÎME, Léona, Ça ! c'est la Martinique, Impr. La Productrice, Fort-de-France, 1966.

HEARN, Lafcadio :

- Esquisses martiniquaises, Mercure de France (trad. fr), Paris, 1924.

- Contes des tropiques, Mercure de France (trad. fr), Paris, 1927.

LECLERC, « Navire Le Siam. Transport de 429 Indiens pris àPondichéry et débarqués à la Martinique », Revue algérienne et coloniale, Paris, 1860, vol. II, p. 105-124.

LEIRIS, Michel, Contacts de civilisations en Martinique et en Guadeloupe, Gallimard / UNESCO, Paris, 1955, rééd. 1987.

LEROY-BEAULIEU, P., De la Colonisation chez les peuples modernes, Guillaumin, Paris, 1874.

LUGSOR, Christiane, L'Immigration indienne à la Martinique, mémoire pour la maîtrise d'histoire, Université Paris X, Paris, 1980.

MEUNIER, Jean-Pierre, livret de : Stellio. Le créateur de la biguine à Paris. Intégrale chronologique, 1929-1931 (Compact Dise), Frémeaux \& Associés, Paris, 1994.

MEUNIER, Jean-Pierre / LÉARDÉE, Brigitte, La Biguine de l'Oncle Ben's. Ernest Léardée raconte, Éditions caribéennes, Paris, 1989.

POITEVIN, Guy / RAIRKAR, Hema, Femmes coolies en Inde. Salariat, culture et survie en ville, Syros, Palis, 1994. 
RENARD, Raymond, La Martinique de 1848 à 1870, Groupe universitaire de recherches inter-caraïbes : Centre universitaire des Antilles et de la Guyane, Paris, no 12, 1973 (1re édition, 1955).

ROMANETTE, Irmine, Sonson de la Martinique, Société d'édition littéraire et technique, Paris, 1932.

SCHELCHER, Victor, Polémique coloniale, rééd. Désormeaux / L'Harmattan, Fort-de-France, 1979 (1ères éditions, de 1871 à 1885), 2 vol.

SINGARAVÉLOU, Les Indiens de la Caraïbe, L'Harmattan, Paris, 1987, 3 vol.

SMERALDA-AMON, La Question de l'immigration indienne dans son environnement socio-économique martiniquais : 1848-1900, L'Harmattan, Paris, 1996.

STELLIO, Alexandre, Stellio. Le créateur de la biguine à Paris. Intégrale chronologique, 1929-1931 (Compact Dise), Frémaux \& Associés, Paris, 1994.

TORABULLY, Khal, « Coolitude », Notre librairie, Paris, 1996, p. 59-71.

\section{Fin du texte}

